\title{
INFECCIÓN NOSOCOMIAL POR ENTEROCOCCUS Hospital de San José, 2005-2007
}

\author{
Edgar Andrés Pulido Gil MD*, Diego Andrés Bonilla González MD*, Andrea Rojas Garzón MD**
}

\section{Resumen}

Objetivos: describir las características epidemiológicas, clínicas, sociodemográficas y desenlace final de los pacientes adultos atendidos en el Hospital de San José con infección nosocomial por Enterococcus sp, los sitios anatómicos más comprometidos, el perfil de sensibilidad antimicrobiana y la presencia de resistencia a la vancomicina, así como determinar la mortalidad global en esta patología. Métodos: analizar los aislamientos positivos de Enterococcus sp en pacientes de 18 años o más, obtenidos de la base de datos LabPro del servicio de microbiología del Hospital de San José entre octubre de 2005 y diciembre de 2007; después seleccionar los aislamientos positivos de origen nosocomial, con base en los criterios establecidos por el CDC de Atlanta. Se recolectaron los datos clínicos, demográficos, y microbiológicos, así como la mortalidad de la población seleccionada. Resultados: de 303 aislamientos positivos para Enterococcus sp 58 fueron nosocomiales y el germen más frecuente fue el Enterococcus faecalis; 56,9\% fueron mujeres y la edad promedio 50 años. Las comorbilidades específicas más comunes fueron hipertensión arterial, enfermedades neoplásicas y diabetes. El $\mathbf{7 4 , 1 \%}$ de los pacientes eran manejados por servicios quirúrgicos y $\mathbf{9 1 , 4 \%}$ del total fueron sometidos a algún procedimiento quirúrgico previo al aislamiento. La mortalidad fue 12\%. Conclusiones: en nuestra población la infección nosocomial por Enterococcus sp corresponde al $19 \%$ del total de positivos para este germen. La especie más frecuente es el Enterococcus faecalis, que puede relacionarse con la alta sensibilidad encontrada a la vancomicina. Las penicilinas y los aminoglucósidos siguen siendo antibióticos de elección pues se identificó una alta sensibilidad en los antibiogramas. Se encontró una elevada resistencia a carbapenémicos, después del quinupristin/dalfopristin, explicado por la alta resistencia intrínseca del Enterococcus faecalis a este grupo antibiótico. La infección nosocomial por Enterococcus sp en el Hospital de San José se relaciona con frecuencia con procedimientos quirúrgicos, en especial los abdominales, lo que sugiere en este tipo de pacientes una mayor predisposición a la infección nosocomial. La mortalidad global fue $\mathbf{1 2} \%$ del total de la muestra y para aquellos que requirieron manejo en la UCI fue del $\mathbf{3 1 , 8 \%}$, pudiendo inferir que una mayor complejidad y comorbilidad influyen en un alto riesgo de desenlace fatal.

Palabras clave: Enterococcus, infección cruzada, resistencia a vancomicina.

Abreviaturas: UCI, unidad de cuidados intensivos.

\section{NOSOCOMIAL INFECTIONS BY ENTEROCOCCI AT THE SAN JOSÉ HOSPITAL}

\section{Abstract}

Objectives: to describe the epidemiological, clinical and socio-demographic features and the final outcome of adult patients diagnosed with a nosocomial infection by Enterococcus sp at the San José Hospital; to identify the most

Fecha recibido: septiembre 4 de 2008 - Fecha aceptado: enero 9 de 2009

Residente III de Medicina Interna, Fundación Universitaria de Ciencias de la Salud, Hospital de San José. Bogotá D.C. Colombia.
Especialista en Medicina Interna, Instructora de Medicina Interna, Fundación Universitaria de Ciencias de la Salud, Hospital de San José. Bogotá D.C. Colombia. 
commonly involved anatomic sites; to describe the antibiotic sensitivity profile and the presence of vancomycin resistance; and to determine the overall mortality rate for this condition. Methods: all positive isolates of Enterococcus sp obtained from the LabPro database of the Department of Microbiology at the San José Hospital, between October 2005 and December 2007, in older than 18 year patients, were analyzed; positive isolates of nosocomial origin were selected based on the CDC criteria. Clinical, demographic and microbiological data, as well as, the mortality rate in the studied population were gathered in each case. Results: out of 303 positive isolates for Enterococcus sp, 58 were nosocomial and Enterococcus faecium was the commonest organism isolated; $56.9 \%$ were female patients and the mean age was 50 years. The most common specific comorbidities were arterial hypertension, neoplastic disease and diabetes. Of all participating patients, $74.1 \%$ were managed in surgical departments and $91.4 \%$ underwent a surgical procedure before the isolation of a microorganism. The mortality rate was $12 \%$. Conclusions: we concluded that in our population, nosocomial infections caused by Enterococcus sp account for $19 \%$ of the total positive isolates for this agent. The most commonly isolated strain is Enterococcus faecium, which can be related to the high sensitivity to vancomycin identified. Due to the high level of sensitivity identified in antibiograms, penicillines and aminoglycosides continue to be the preferred antibiotics to treat these infections. In addition, we found a high resistance to carbapenemics, after administering quinupristin/dalfopristin, explained by the high intrinsic resistance of Enterococus faecium to this group of antibiotics. Nosocomial infection by Enterococcus $s p$ at the San José Hospital is frequently related to surgical procedures especially abdominal procedures, which suggests that these patients have a greater predisposition to nosocomial infection. Overall mortality rate was $12 \%$ on the total sample and $31.8 \%$ on patients transferred to the ICU. It can be inferred that greater complexity and co-morbidity influence a high risk of fatal outcome.

Key words: Enterococcus, crossed infection, vancomycin resistance.

\section{Introducción}

Las infecciones nosocomiales se han convertido en importantes causas de mortalidad en los últimos años. . $^{1,2,3,4,5}$ Su impacto no es sólo clínico, ya que se ha estimado que el costo en los Estados Unidos asciende a 4.500 millones de dólares anuales. ${ }^{6} \mathrm{El}$ auge de nuevos métodos diagnósticos y terapéuticos invasivos, el uso de antibióticos de amplio espectro, la mayor estancia hospitalaria, el manejo en UCI, así como la supervivencia de pacientes con patologías de mayor complejidad y comorbilidad, han sido descritos a nivel mundial como factores de riesgo para el desarrollo de infecciones nosocomiales y para el surgimiento de cepas multirresistentes que reducen las posibilidades terapéuticas y pronósticas en este tipo de enfermos. ${ }^{5}$

Dentro de los gérmenes causales de infección nosocomial, además de Gramnegativos multirresistentes, ha cobrado gran importancia el grupo de Grampositivos tipo Enterococcus sp, así como el desarrollo de resistencia a antibióticos de mayor espectro. El Enterococcus $s p$ ha pasado de agente comensal de escasa patogenicidad a ser la tercera etiología más común de infección nosocomial. ${ }^{6,78,9} \mathrm{Si}$ se encuentra ese comportamiento en nuestra población, puede llegar a constituir no sólo una problemática intrainstitucional sino un problema de salud pública.

En los últimos años a consecuencia del aumento de la incidencia global de infección nosocomial por Enterococcus $s p,,^{2,3}$ han surgido diferentes estudios médicos a nivel mundial que describen su comportamiento y una serie de factores de riesgo que facilitan el desarrollo de infecciones intrahospitalarias por este germen. Dentro de estos factores se encuentran los pacientes sometidos a algún procedimiento quirúrgico, aquellos con enfermedades concomitantes (neoplasias, quemaduras o inmunosupresión), edad avanzada, hospitalización larga en UCI, terapia respiratoria prolongada con ventilación mecánica y antibioticoterapia previa. Aunque según los reportes mundiales el Enterococcus sp no ha llegado a ser el primer agente causal de infección nosocomial, su creciente incidencia en los últimos años y así mismo el desarrollo de una mayor resistencia y mortalidad, obligan a estudiar el comportamiento de dicha infección en nuestra población. . $^{1,2,3,4,5,6,7,8,9}$ 
A pesar de todo lo anterior y de la importancia de este fenómeno, no ha sido informado el comportamiento epidemiológico de este germen en nuestra población. En Colombia sólo existe un estudio que describe un primer brote de infección nosocomial por Enterococcus faecium resistente a vancomicina en la ciudad de Medellín, el cual mostró como factores de riesgo la presencia de portadores en pacientes o trabajadores de la salud, las deficientes prácticas en el aislamiento de contactos y el uso de antimicrobianos como metronidazol, ceftazidima, cefotaxime e imipenem. ${ }^{10}$

En el Hospital de San José, donde durante los últimos años se han incrementado los pacientes de mayor complejidad y comorbilidad, se usan cada vez más antibióticos de amplio espectro (ej.: cefepime, carbapenémicos) y hay mayor estancia hospitalaria en UCI. No se cuenta con estudios que describan las características epidemiológicas que permitan conocer el comportamiento de la infección nosocomial por Enterococcus $s p$, con el fin de poder establecer políticas de prevención y evitar así el desarrollo de brotes de cepas resistentes. Ante la evidencia disponible se realizó este estudio con el fin de profundizar en el conocimiento que se tiene de la epidemiología de nuestra población y del comportamiento de estas infecciones desde el punto de vista de la presencia de comorbilidades, resistencia antimicrobiana, desenlace final del paciente y mortalidad global.

\section{Métodos}

Se trata de un estudio descriptivo retrospectivo tipo serie de casos, en el cual se seleccionaron los aislamientos positivos para Enterococcus registrados en la base de datos LabPro del laboratorio del Hospital de San José en el período comprendido entre octubre de 2005 y diciembre de 2007, obteniendo 401 en adultos, correspondientes a 345 historias clínicas de las cuales se obtuvieron datos completos en $303 \mathrm{ca}$ sos. Después se seleccionaron los que correspondían a infección nosocomial y se les aplicó el instrumento de recolección de datos obteniendo 58 casos para este estudio. Se incluyeron pacientes adultos de 18 años o más, con aislamiento de Enterococcus en cualquier sitio anatómico y que presentaron signos de respuesta inflamatoria sistémica o sepsis de acuerdo con lo establecido en la Conferencia de Consenso Internacional para definiciones en sepsis ${ }^{11}$ o que tuvieran signos de infección localizada como ocurre en la mayoría de infecciones del sitio operatorio. ${ }^{12,13}$ Para clasificar la infección como nosocomial se aplicaron los criterios que en este sentido tiene el Center for Disease Control and Prevention de Atlanta. ${ }^{14}$ No se incluyeron en el estudio pacientes con diagnóstico de infección por Enterococcus adquirida en la comunidad, en otras instituciones o que lo fueron en el Hospital de San José pero que no tuvieron el seguimiento completo por remisión del paciente a otra institución. Para cumplir con los objetivos de este estudio se utilizaron medidas estadísticas descriptivas (porcentajes, medidas de tendencia central y de dispersión). Todos los análisis se realizaron en el programa estadístico STATA 10.0 (StataCorp LP College Station TX 77845 USA versión 10.0 para Windows).

\section{Resultados}

Características demográficas: se analizaron 58 casos correspondientes a infección intrahospitalaria por Enterococcus sp (19\% del total). De las nosocomiales el $56,9 \%$ correspondieron a mujeres. La edad promedio fue de 50 años (DE: 19,2), con una mínima de 18 y máxima de 87 años, para una mediana de 49.

Características clínicas: la mediana de estancia hospitalaria fue de 14.5 días con un mínimo de dos y un máximo de 165 días. La mediana previa al aislamiento fue de 3 días. La frecuencia de comorbilidades en la población objeto del estudio se define en la Tabla 1. La mayoría de casos se presentaron en los servicios quirúrgicos con una frecuencia de $43(74,1 \%)$, seguido por medicina interna con nueve casos $(15,5 \%)$ y por último ginecología y obstetricia con seis casos (10,3\%). La frecuencia según la subespecialidad se muestra en la Tabla 2. Los procedimientos quirúrgicos previos al aislamiento se llevaron a cabo en 53 pacientes $(91,4 \%)$, la mayoría de los cuales fueron abdominales $(37,7 \%)$ (Gráfico 1). 


\section{Tabla I. Frecuencia de comorbilidades con infección nosocomial por Enterococcus}

\begin{tabular}{|l|c|c|}
\hline Comorbilidad & Frecuencia & Porcentaje \\
\hline Neoplasias & 7 & 12 \\
\hline Diabetes & 4 & 6,9 \\
\hline Enfermedades del colágeno & 4 & 6,9 \\
\hline EPOC & 2 & 3,5 \\
\hline Ingesta de corticoides & 2 & 3,5 \\
\hline Neutropenia febril & 2 & 3,5 \\
\hline Insuficiencia renal crónica & 1 & 1,7 \\
\hline VIH/SIDA & 1 & 1,7 \\
\hline Insuficiencia cardíaca & 1 & 1,7 \\
\hline HTA & 17 & 29,3 \\
\hline Otros (obesidad, hipotiroidismo, etc.) & 17 & 29,3 \\
\hline Ninguna & 27 & 46 \\
\hline
\end{tabular}

Tabla 2. Frecuencia de aislamiento nosocomial de Enterococcus según subespecialidad

\begin{tabular}{|l|c|c|}
\hline Subespecialidad & Frecuencia & Porcentaje \\
\hline Cx general & 14 & 24,1 \\
\hline Ortopedia & 9 & 15,5 \\
\hline Neurocirugía & 6 & 10,3 \\
\hline Vascular & 5 & 8,6 \\
\hline Urología & 4 & 6,9 \\
\hline Medicina interna & 4 & 6,9 \\
\hline Sépticas & 3 & 5,2 \\
\hline Cirugía plástica & 3 & 5,2 \\
\hline Ginecoobstetricia & 2 & 3,5 \\
\hline Hematología & 2 & 3,5 \\
\hline Neurología & 2 & 3,5 \\
\hline Oncología & 1 & 1,7 \\
\hline Cx de tórax & 1 & 1,7 \\
\hline Ginecología oncológica & 1 & 1,7 \\
\hline Cardiovascular & I & 1,7 \\
\hline TOTAL & 58 & 100 \\
\hline
\end{tabular}




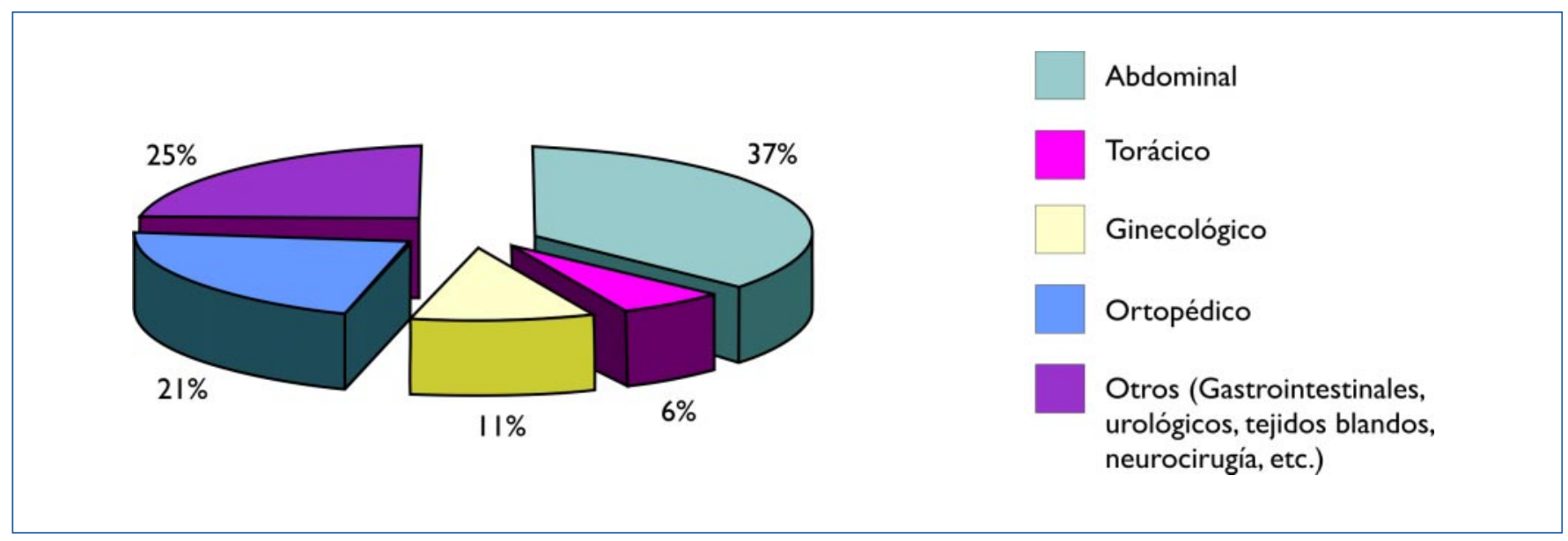

Gráfico I. Frecuencia de procedimiento quirúrgico por localización anatómica antes del aislamiento.

Un total de 22 pacientes (38\%) cursaron con compromiso ventilatorio previo con frecuente falla ventilatoria y $15(25 \%)$ con afección neurológica, en su mayoría por alteración del estado de alerta con una escala de coma de Glasgow menor de 8. En relación con la necesidad de UCI previa al aislamiento se encontró que 22 enfermos $(38 \%)$ requirieron ingreso a este servicio, con una mortalidad del 31,8\% (7 pacientes) para una mortalidad global del $12 \%$. De los pacientes fallecidos tres $(42,8 \%)$ cursaron con bacteremia y de los cuatro restantes dos casos correspondieron a infección urinaria sin bacteremia, uno presentó neumonía asociada con ventilador y otro infección del sitio operatorio. Todos murieron en UCI.
Características del aislamiento: la fuente más común de aislamiento fue la secreción de la herida quirúrgica, como se ve en la Gráfica 2. De los 581 casos, 53 (91,3\%) se encontraban recibiendo tratamiento antibiótico al momento del aislamiento. En este grupo el fármaco previo más utilizado fue ampicilina sulbactam (34\%), seguido de cefazolina y vancomicina; 35 casos $(60 \%)$ recibieron dos o más antibióticos (Tabla 3). En promedio cada enfermo recibió dos antibióticos diferentes durante el curso de su estancia hospitalaria (2.68; DE: 1.76). Los datos del tratamiento farmacológico posterior al aislamiento muestran que el $81,1 \%$ de los pacientes continuó con manejo antibiótico, siendo también ampicilina sulbactam

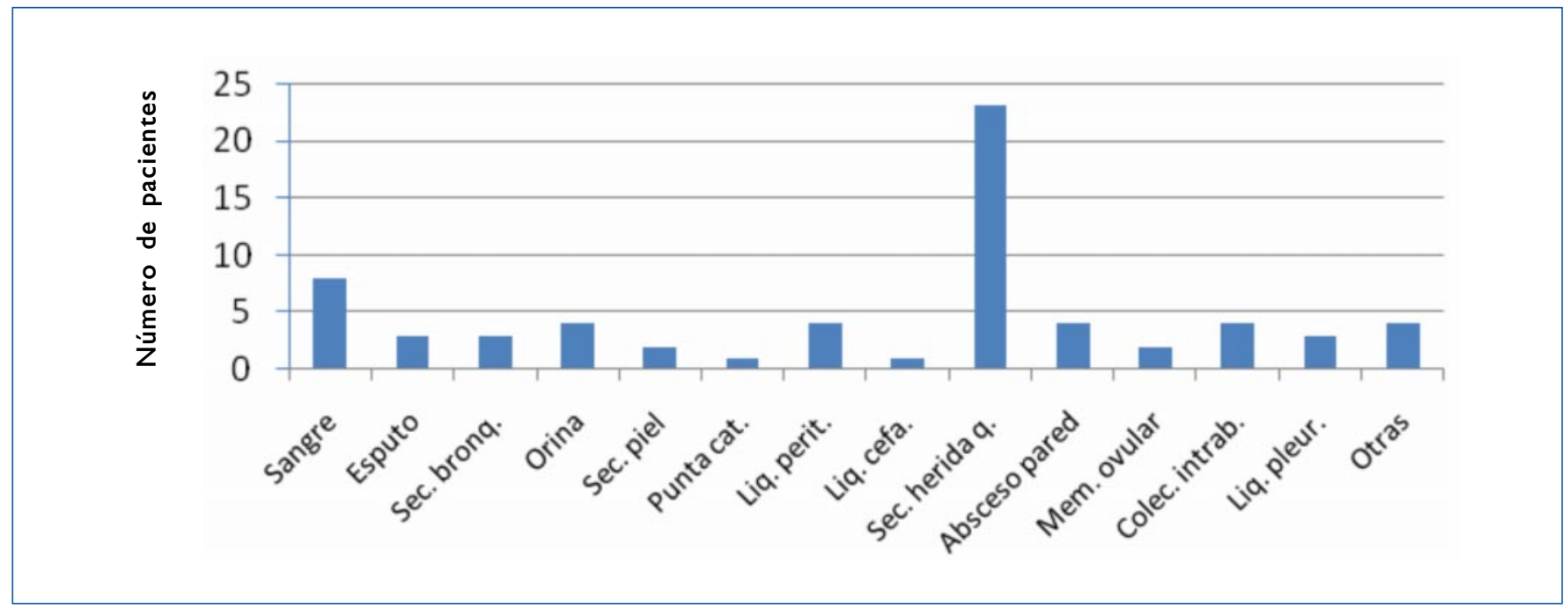

Gráfico 2. Distribución de los pacientes según el sitio de aislamiento. 


\begin{tabular}{|c|c|c|c|c|c|c|}
\hline Antibiótico previo al aislamiento & Frecuencia & Porcentaje & Días promedio & Mediana & Mínimo & Máximo \\
\hline Ampicilina/sulbactam & 18 & 33.9 & 4.77 & 3 & 1 & 17 \\
\hline Cefazolina & 17 & 32.1 & 3.1 & 1.5 & 1 & 12 \\
\hline Vancomicina & 10 & 18.9 & 6.7 & 4.5 & 1 & 27 \\
\hline Clindamicina & 10 & 18.9 & 4.25 & 2 & 1 & 13 \\
\hline Oxacilina & 9 & 16.9 & 4.14 & 2 & I & 18 \\
\hline Piperacilina/tazobactam & 9 & 16.9 & 3.88 & 1 & 1 & 14 \\
\hline Gentamicina & 8 & 15.1 & 5.75 & 3.5 & 1 & 18 \\
\hline Meropenem & 7 & 13.2 & 4.5 & 3 & 1 & 17 \\
\hline Cefepime & 7 & 13.2 & 4.33 & 2 & 1 & 10 \\
\hline Amikacina & 6 & 11.3 & 3.4 & 2 & 1 & 8 \\
\hline Imipenem & 4 & 7.5 & 8.75 & 11 & 1 & 12 \\
\hline Ceftriaxona & 4 & 7.5 & 1.5 & 1 & I & 3 \\
\hline Metronidazol & 3 & 5.6 & 3 & 3 & I & 5 \\
\hline Penicilina & 2 & 3.7 & 1 & 1 & 1 & 1 \\
\hline Claritromicina & 2 & 3.7 & 6.5 & 6.5 & 3 & 10 \\
\hline Cefalotina & 2 & 3.7 & 3 & 3 & 3 & 3 \\
\hline Cefalexina & I & 1.9 & 7 & 7 & 7 & 7 \\
\hline
\end{tabular}

* Nota: $n=53$.

el más frecuente seguido por vancomicina y otros de amplio espectro como piperacilina tazobactam y carbapenémicos (Tabla 4). En 41 casos (70,6\%) hubo cambio de por lo menos un antibiótico después del aislamiento.

Características microbiológicas: la especie aislada con mayor frecuencia fue Enterococcus faecalis $(94,8 \%$ de los casos), seguida de Enterococcus faecium (3,45\%) y Enterococcus gallinarum (1,72\%). En cuanto al perfil de sensibilidad en el antibiograma, no se encontró ninguna cepa resistente a vancomicina y la resistencia a penicilinas y aminoglucósidos fue mínima en contraste con una elevada a carbapenémicos, después del quinupristin/dalfopristin, explicado en la alta resistencia intrínseca del Enterococcus faecalis a este grupo antibiótico (Tabla 5).

\section{Discusión}

Los resultados del presente estudio indican que la especie más frecuente aislada en los casos de infección nosocomial por Enterococcus sp en nuestra institución es el Enterococcus faecalis, lo cual se correlaciona con la información suministrada por otros estudios a nivel mundial. ${ }^{1,2,3,4,5}$ Desde el punto de vista demográfico existe un leve compromiso mayor en las mujeres con alta frecuencia en adultos de edad media, lo que contrasta con los datos obtenidos a nivel mundial en donde los mayores de 65 años tienen una predisposición superior a adquirir este tipo de infección. ${ }^{15,16}$ La diferencia que encontramos en este estudio, podría explicarse por la elevada frecuencia de infección del sitio operatorio en cirugía ortopédica por trauma, que ocurre en especial en pacientes jóvenes. 


\begin{tabular}{|l|c|c|}
\hline \multicolumn{2}{|c|}{ Tabla 4. Antibiótico administrado después del aislamiento* } \\
\hline Antibiótico posterior al aislamiento & Frecuencia & Porcentaje \\
\hline Ampicilina/sulbactam & 11 & 25,5 \\
\hline Vancomicina & 10 & 23,2 \\
\hline Piperacilina/tazobactam & 8 & 18,6 \\
\hline Imipenem & 6 & 13,9 \\
\hline Meropenem & 6 & 13,9 \\
\hline Gentamicina & 5 & 11,6 \\
\hline Oxacilina & 5 & 11,6 \\
\hline Cefepime & 4 & 9,3 \\
\hline Ceftriaxona & 4 & 9,3 \\
\hline Clindamicina & 4 & 9,3 \\
\hline Cefazolina & 3 & 6,9 \\
\hline Metronidazol & 1 & 2 \\
\hline Amikacina & 1 & 2 \\
\hline
\end{tabular}

* Nota: $n=43$.

\begin{tabular}{|l|c|c|c|c|}
\hline \multicolumn{5}{|c|}{ Tabla 5. Porcentaje de sensibilidad in vitro según el antibiótico } \\
\hline Antibiótico & Sensible & Intermedio & Resistente & No hay datos \\
\hline Vancomicina & 100 & 0 & 0 & 0 \\
\hline Penicilinas & 94.83 & 0 & 3.45 & 1.72 \\
\hline Quinolonas & 77.59 & 1.72 & 10.34 & 10.34 \\
\hline Carbapenémicos & 62.07 & 0 & 37.93 & 0 \\
\hline Quinopristin/dalfopristin & 1.72 & 3.45 & 89.66 & 5.17 \\
\hline Aminoglucósidos & 86.21 & 0 & 8.62 & 5.17 \\
\hline
\end{tabular}

Al analizar los factores de riesgo para la infección nosocomial por Enterococcus sp descritos en la literatura, como la estancia hospitalaria prolongada, , 1,2,3,4,5,6,7,8,9 se encontró que en nuestra población ésta fue de 14.5 días, siendo significativamente mayor al promedio general hospitalario de 3.4 días. Otro factor predisponente descrito es la estancia previa en UCI, factor que estuvo presente en $38 \%$ de nuestros pacientes. Por otra parte, ciertas comorbilidades que en su mayoría se relacionan con el estado de depresión inmunológica del paciente $\mathrm{e}^{1,2,3,4,5,6,7,8,9}$ no ocuparon en nuestra población un lugar relevante y en su defecto se encontró como mayor comorbilidad la patología cardiovascular representada en su mayoría por hipertensión arterial ${ }^{16}$ seguida por enfermedades neoplásicas y diabetes. Además, el compromiso ventilatorio o neurológico previo se encontró en menos del $40 \%$.

Nuestros datos señalan una posible asociación de la infección con el antecedente de realización de procedimientos invasivos, asociación informada en la literatu$\mathrm{ra}^{3,4,5}$ lo cual se refleja en la mayor frecuencia de casos en los servicios quirúrgicos, sobre todo en cirugía general. Puede explicarse por el alto número de cirugías a 
nivel abdominal, siendo éste el hábitat natural del Enterococcus sp. Lo anterior también explica la mayor frecuencia de aislamientos en la secreción de la herida quirúrgica (38\%), sumado a que el Enterococcus sp puede sobrevivir en objetos inanimados durante mucho tiempo. ${ }^{17}$

Con respecto al uso de antibióticos antes del aislamiento microbiológico, se puede concluir que los antimicrobianos más usados en nuestra institución en los pacientes con infección nosocomial por Enterococcus sp son la ampicilina sulbactam, la cefazolina, la vancomicina y la clindamicina por más de tres días, lo cual difiere de los datos del estudio realizado en el Hospital San Vicente de Paúl. ${ }^{10}$ Estas diferencias muestran la variabilidad que existe entre los centros hospitalarios en cuanto a la población que es atendida y a la utilización de antibióticos por parte del personal médico. La importancia de este hallazgo radica en la predisposición a infección nosocomial que conlleva el uso de estos fármacos. ${ }^{10}$

En cuanto al empleo de antibióticos para el manejo de la infección nosocomial por Enterococcus sp, continúan en los primeros lugares de preferencia médica la ampicilina sulbactam y la vancomicina, seguidas por antibióticos de amplio espectro como la piperacilina tazobactam y los carbapenémicos. Llama la atención que a pesar de la alta sensibilidad antibiótica del germen, el uso de vancomicina y antibióticos de amplio espectro es frecuente, y aunque en algunos casos se justifica por la estancia en la UCI y al aislamiento de otros gérmenes en este tipo de pacientes, debe tenerse prudencia pues este comportamiento facilitaría el desarrollo de resistencia antibiótica a vancomicina, que hasta el momento no se ha identificado en nuestra población. ${ }^{10}$

Es así como la sensibilidad a la vancomicina fue del $100 \%$, hallazgo que puede relacionarse con una mayor frecuencia de Enterococcus faecalis en los aislamientos, cuyo comportamiento natural es la menor tendencia a desarrollar resistencia a los glucopéptidos en comparación con el Enterococcus faecium, especie de la que sólo se obtuvieron dos aislamientos.

Por otra parte, se encontró una elevada resistencia a carbapenémicos, después del quinupristin/dalfopristin, explicado por la alta resistencia intrínseca del Enterococcus faecalis a este grupo antibiótico. En con- traste, el quinupristin/dalfopristin tiene entre sus indicaciones el tratamiento de infecciones por Enterococcus faecium multirresistente. ${ }^{18}$

En nuestra población de estudio la mortalidad global fue del $12 \%$, que es menor con respecto a la reportada a nivel mundial. ${ }^{1,5}$ Todos los pacientes fallecidos requirieron manejo en la UCI explicado por su gran complejidad. La mayoría de éstos tuvieron bacteremia, lo cual implica un alto compromiso sistémico y un riesgo de mortalidad, que se correlaciona con la información obtenida en la literatura mundial. ${ }^{1,2,3,4,5,6,7,8,9}$ Se puede considerar que el riesgo de mortalidad es de origen multifactorial en estos casos.

Este estudio contó con algunas deficiencias que deben ser contempladas. En primer lugar, se desconoce si hubo falta de registros en las bases de datos de estadística o de bacteriología de algún paciente con infección intrahospitalaria por Enterococcus sp. Tampoco se lograron revisar la totalidad de las historias de los pacientes con aislamientos positivos debido a que no se encontraban en los archivos de estadística del hospital, por ello los datos pueden no representar el cien por ciento de lo ocurrido con este tipo de pacientes en la población estudio. Para controlar este error se realizó una revisión sistemática de tales registros y se verificaron en varias oportunidades las bases de datos del área de microbiología del laboratorio. A pesar de ello, este sesgo no se puede controlar por completo. Una debilidad inherente al tipo de estudio es que estos resultados no permiten establecer factores de riesgo para infección nosocomial por Enterococcus, pues para ello se necesitaría un estudio prospectivo.

Otra deficiencia importante que pudimos constatar mientras se realizaba la recolección de datos es que no se contemplaron los aislamientos concomitantes en estos pacientes, hecho que se daba con bastante frecuencia, ya que podía alterar el curso clínico y el tratamiento instaurado.

La principal fortaleza de este estudio es que permitió profundizar en el conocimiento que se tiene de la epidemiología de nuestra población y del comportamiento de estas infecciones. Es posible que los resultados del estudio sean útiles para otras instituciones donde se manejen pacientes similares. Así mismo, puede servir como base 
para el desarrollo de estudios prospectivos con mayor poder estadístico que permitan determinar escalas de riesgo para la adquisición de infección nosocomial por Enterococcus sp, establecer patrones de susceptibilidad antibiótica, definir factores de riesgo para resistencia antibiótica y mortalidad, mejorar políticas de prevención y de disminución de morbimortalidad.

\section{Referencias}

1. Patterson JE, Sweeney AH, Simms M, Carley N, Mangi $\mathrm{R}$, Sabetta J, et al. An analysis of 110 serious enterococcal infections: epidemiology, antibiotic susceptibility, and outcome. Medicine (Baltimore). 1995; 74 (4): 191-200.

2. Landry SL, Kaiser DL, Wenzel RP. Hospital stay and mortality attributed to nosocomial enterococcal bacteremia: a controlled study. Am J Infect Control.1989; 17:323-9.

3. Pallarés R, Barberá MJ, Guillamont J. Bacteriemia enterocócica nosocomial. Rev Clin Esp. 1995; 195:12-5.

4. Maki DG, Agger WA. Enterococcal bacteremia: clinical features, the risk of endocarditis, and management. Medicine (Baltimore). 1988; 67:248-69.

5. Pallarés R, Pujol M, Peña MC, Ariza J, Martín R, Gudiol F. Cephalosporins a risk factor for nosocomial enterococcus faecalis bacteremia: a matched case-control study. Arch Intern Med. 1993; 153:1581-6.

6. Wisplinghoff H, Bischoff T, Tallent SM, Seifert H, Wenzel RP, Edmond MB. Nosocomial bloodstream infections in US hospitals: analysis of 24,179 cases from a prospective nationwide surveillance study. Clin Infect Dis. 2004; 39:309-17.

7. Tailor SA, Bailey EM, Rybak MJ. Enterococcus, an emerging pathogen. Ann Pharmacother. 1993; 27:1231-42.

8. Moellering RC. Emergence of enterococcus as a significant pathogen. Clin Infect Dis. 1992; 14:1173-6.
9. Cartón Sánchez JA. Epidemiología de las infecciones enterocócicas. Rev Clin Esp. 1995; 195:3-11.

10. Ospina S, Robledo J, Gómez CI, et al. Infección nosocomial por enterococo resistente a la vancomicina: características clínico epidemiológicas y factores de riesgo. Infection. 2001; 5:14-20.

11. Levy MM, Fink MP, Marshall JC, Abraham E, Angus D, Cook D, Cohen J, Opal SM, Vincent JL, Ramsay G. $2001 \mathrm{SCCM} / \mathrm{ESICM} / \mathrm{ACCP} / \mathrm{ATS} / \mathrm{SIS}$ International Sepsis Definitions Conference. Crit Care Med. 2003; 31(4):1250-6.

12. Barie PS, Eachempati SR. Surgical Site Infections. Surg Clin N Am. 2005; 85:1115-35.

13. Calandra T, Cohen J. The international sepsis forum consensus conference on definitions of infection in the intensive care unit. Crit Care Med. 2005; $33: 1538-48$

14. Horan TC, Gaynes RP. Surveillance of nosocomial infections. En: Mayhall CG. Hospital Epidemiology and Infection Control. 3rd ed. Philadelphia: Lippincott Williams \& Wilkins; 2004. p. 1672-89.

15. Martínez-Odriozola $P$, Muñoz-Sánchez J, GutiérrezMacías A, Arriola-Martínez P, Montero-Aparicio E, Ezpeleta-Baquedano C, et al. Análisis de 182 episodios de bacteriemia por enterococo: estudio de la epidemiología, microbiología y evolución clínica. Enferm Infecc Microbiol Clin. 2007; 25(8):503-7.

16. Lavigne JP, Marchandin H, Czarnecki E, Kaye C, Sotto A. Bactériémies à Enterococcus spp.:etude prospective au CHU de Nîmes Enterococcal bacteremia at Nîmes university hospital. Pathol Biol (Paris). 2005; 53 (8-9): $539-45$.

17. Garner JS. Guideline for isolation precautions in hospitals. The Hospital Infection Control Practices Advisory Committee. Infect Control Hosp Epidemiol. 1996; 17(1):53-80.

18. Zirakzadeh A, Patel R. Vancomycin-resistant enterococci: colonization, infection, detection, and treatment. Mayo Clin Proc. 2006; 81(4):529-36. 